\title{
The Estimation of Open Boundary Conditions in the Bohai Sea with POD 4D VAR Data Assimilation
}

\author{
Shouguo Qian ${ }^{1,2}$, Xianqing $\mathrm{Lv}^{3}$, Rencheng $\mathrm{Sun}^{4}$ and Fengjing Shao ${ }^{1,4, *}$ \\ ${ }^{1}$ Complexity Science Research Institute, Qingdao University, Qingdao 266071, China \\ ${ }^{2}$ School of Mathematical Sciences, Qingdao University, Qingdao 266071, China \\ ${ }^{3}$ Laboratory of Physical Oceanography, Ocean University of China, Qingdao 266100, China \\ ${ }^{4}$ College of Computer Science \& Technology, Qingdao University, Qingdao 266071, China \\ ${ }^{*}$ Corresponding author
}

\begin{abstract}
Proper orthogonal decomposition four-dimensional Variational (POD 4D VAR) data assimilation method is used to estimate the open boundary conditions (OBCs) with a twodimensional (2D) $M_{2}$ tide in the Bohai Sea by assimilating the TOPEX/Poseidon (T/P) altimeter data. Qian et al. [14] assume that the OBCs are temporally and spatially varying and optimize the OBCs in the Bohai, Yellow and East China Seas and in this paper we only optimize the Fourier coefficients of the OBCs. In the practical experiment, the absolute mean difference between the model results and observations are $2.8 \mathrm{~cm}$ and $2.3^{\circ}$ for amplitude and phase-lag, respectively, and the cochart obtained can describe the character of $\mathrm{M}_{2}$ tide in the Bohai Sea, suggesting that the inversion of the OBCs is successful.
\end{abstract}

Keywords-proper orthogonal decomposition; four-dimensional Variationl; data assimilation; open boundary conditions; TOPEX/Poseidon altimeter data

\section{INTRODUCTION}

Open boundary conditions (OBCs) are crucial for the representation of tidal processes in the shallow water model which are needed to be adjusted by compared with model results and observations. The 4D VAR data assimilation method is one of powerful tool for parameter estimation ([19]). Zhang and Lv [10-11] estimated OBCs or the bottom friction coefficient in different models.

One problem of the 4D VAR data assimilation is characterized by the great CPU time and memory load required for the computation of the cost function and its gradient. To solve this problem, some reduced order methods of high dimensional or infinite dimensional hydrodynamic model have been proposed. Among them, POD 4D VAR data assimilation method had also been successfully used for oceanic problems (Du et al. [12] and Cao et al. [13]).

Qian et al. [14] had tested the feasibility of the POD reduced order model and assume that the OBCs are temporally and spatially varying and optimize the OBCs in the Bohai Sea, the

Yellow Sea and the East China Sea. In this paper, we only optimize the Fourier coefficients of the OBCs. In the practical experiment, the absolute mean difference between the simulation result and observations are $2.8 \mathrm{~cm}$ and $2.3^{\circ}$ in amplitude and phase-lag and the Co-tidal charts obtained can describe the character of $\mathbf{M}_{2}$ tide in the Bohai Sea.

This article is organized as follows: The optimization of the OBCs is described in Section 2; the numerical experiment and results are listed in Section 3; we make a summary and draw some conclusions in Section 4.

\section{THE OPTIMIZATION OF THE OBCS}

In this paper, the 2D tidal model and its POD reduced order method is the same as the constructed by Qian et al. [14] which contains the infinite difference of the 2D tidal model, the POD reduced order forward and adjoint model. The POD reduced order forward model is used to simulate the tides and the adjoint model is used to optimize the control variables. We assume that $(m, n)$ belonging to the open boundary, and the OBCs are respected as

$$
\zeta_{m, n}^{j}=a_{m, n} \cos (\omega j \Delta t)+b_{m, n} \sin (\omega j \Delta t)
$$

where $a_{m, n}, b_{m, n}$ are the Fourier coefficients. The optimization of the OBCs is listed as follows.

$$
\begin{aligned}
& \operatorname{If}(m, n) \in S_{u}, \text { then } \\
& \frac{\partial J}{\partial a_{m, n}}=-\sum_{j=1}^{2 L_{t}} \sum_{l=1}^{L_{u}} \frac{\eta_{l}^{j} \phi_{l, m, n}^{u} \Delta \operatorname{tg} \cos (\omega j \Delta t)}{\Delta x_{n}}, \\
& \frac{\partial J}{\partial b_{m, n}}=-\sum_{j=1}^{2 L_{t}} \sum_{l=1}^{L_{u}} \frac{\eta_{l}^{j} \phi_{l, m, n}^{u} \Delta \operatorname{tg} \sin (\omega j \Delta t)}{\Delta x_{n}} . \\
& \operatorname{If}(m-1, n) \in S_{u} \text {, then } \\
& \frac{\partial J}{\partial a_{m, n}}=-\sum_{j=1}^{2 L_{t}} \sum_{l=1}^{L_{u}} \frac{\eta_{l}^{j} \phi_{l, m-1, n}^{u} \Delta \operatorname{tg} \cos (\omega j \Delta t)}{\Delta x_{n-1}}, \\
& \frac{\partial J}{\partial b_{m, n}}=-\sum_{j=1}^{2 L_{t}} \sum_{l=1}^{L_{u}} \frac{\eta_{l}^{j} \phi_{l, m-1, n}^{u} \Delta \operatorname{tg} \sin (\omega j \Delta t)}{\Delta x_{n-1}} . \\
& \text { If }(m, n) \in S_{v}, \quad \text { then }
\end{aligned}
$$




$$
\begin{array}{r}
\frac{\partial J}{\partial a_{m, n}}=-\sum_{j=1}^{2 L_{l}} \sum_{l=1}^{L_{v}} \frac{\mu_{l}^{j} \phi_{l, m, n}^{v} \Delta \operatorname{tg} \cos (\omega j \Delta t)}{\Delta y}, \\
\frac{\partial J}{\partial b_{m, n}}=-\sum_{j=1}^{2 L_{h}} \sum_{l=1}^{L_{v}} \frac{\mu_{l}^{j} \phi_{l, m, n}^{v} \Delta t g \sin (\omega j \Delta t)}{\Delta y} . \\
\text { If }(m, n-1) \in S_{v} \text {, then } \\
\frac{\partial J}{\partial a_{m, n}}=-\sum_{j=1}^{2 L_{L}} \sum_{l=1}^{L_{v}} \frac{\mu_{l}^{j} \phi_{l, m, n-1}^{v} \Delta \operatorname{tg} \cos (\omega j \Delta t)}{\Delta y}, \\
\frac{\partial J}{\partial b_{m, n}}=-\sum_{j=1}^{2 L_{L}} \sum_{l=1}^{L_{v}} \frac{\mu_{l}^{j} \phi_{l, m, n-1}^{v} \Delta t g \sin (\omega j \Delta t)}{\Delta y} .
\end{array}
$$

If $(m, n)$ belongs to the above a variety of situations, the gradient of the cost function with respect to the OBC is equal to the sum of the corresponding gradients. The OBCs are adjusted as follows:

$$
\begin{gathered}
\hat{a}_{m, n}=a_{m, n}-\rho_{a_{m, n}} \frac{\partial J}{\partial a_{m, n}}, \\
\hat{b}_{m, n}==b_{m, n}-\rho_{b_{m, n}} \frac{\partial J}{\partial b_{m, n}},
\end{gathered}
$$

where $\rho$ is the step length and $\hat{a}_{m, n}, \hat{b}_{m, n}$ are the updated values of $a_{m, n}, b_{m, n}$, respectively. The optimization steps are as same as [14].

\section{NUMERICAL EXPERIMENT AND RESULTS}

In the present study, the computing area is the Bohai sea $\left(117.5^{\circ} \mathrm{E}-122.5^{\circ} \mathrm{E}, 37^{\circ} \mathrm{N}-41^{\circ} \mathrm{N}\right.$, Figure 1). From Figure 1, we can find that its mean depth is $18 \mathrm{~m}$, and the deepest point is located in the west of Lao Tie Shan channel. The OBCs and T/P satellite tracks are also shown in Figure 1. The horizontal resolution is $10^{\prime} \times 10^{\prime}$. From The angular frequency of $\mathrm{M}_{2}$ tide is $1.405189 \times 10^{-4} \mathrm{~s}^{-4}$ and the time step is $372.618 \mathrm{~s}(1 / 120$ of the period of $\mathrm{M}_{2}$ tide). The initial conditions are $\zeta=u=v=0$ at $t=0$. The closed boundary condition is that the normal velocity is zero. The bottom friction coefficients are set as 0.002 and the assigned Fourier coefficients $a_{m, n}, b_{m, n}$ are taken as zero.

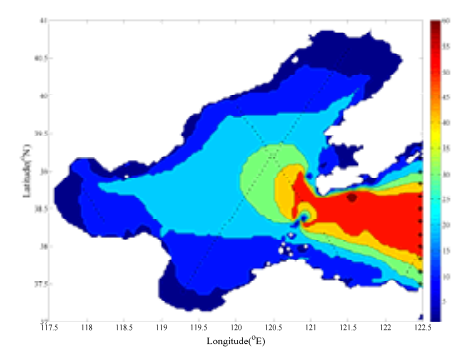

FIGURE I. THE BATHYMETRIC MAP OF BOHAI SEA, THE POSITION OF T/P SATELLITE TRACKS, AND THE OBCS. THE SOLID LINES ARE T/P SATELLITE TRACKS. THE SYMBOL '+' STANDS FOR OBC

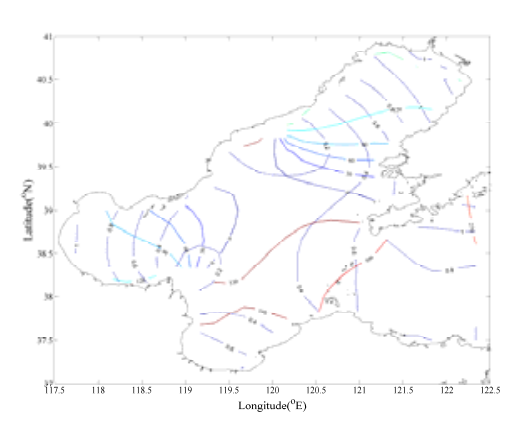

FIGURE II. THE COMPUTED CO-TIDAL CHARTS OF $\mathrm{M}_{2}$ TIDE. THE DOTTED AND SOLID LINES DENOTE THE AMPLITUDE AND PHASE-LAG (DEG), RESPECTIVELY

The absolute mean difference of amplitude and phase-lag are $2.8 \mathrm{~cm}$ and $2.3^{\circ}$, respectively. The results of the experiment in this paper appear to coincide with the observed $\mathrm{M}_{2}$ tide in the Bohai Sea fairly well (Figure 2). The results of the absolute mean difference and Figure 2 are satisfactory and indicate that the observations have been successfully assimilated into the model.

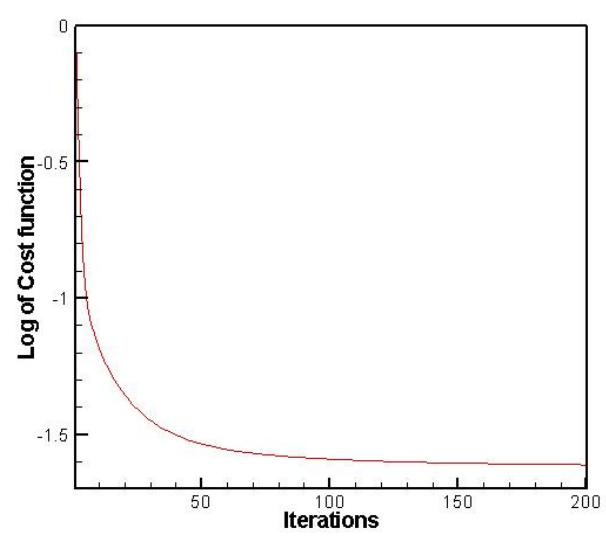

FIGURE III. THE VALUES $J / J_{0}$ VERSUS ITERATION NUMBER,

$$
\begin{gathered}
\text { WHERE }{ }^{J_{0}} \text { REPRESENTS THE INITIAL VALUE OF THE COST } \\
\text { FUNCTION }
\end{gathered}
$$

The cost function and its gradients with respect to the OBCs are two important convergence criteria for data assimilation in this model. Figure 3 shows the cost values $J$ (normalized by its initial value ${ }^{J_{0}}$ ). We define the gradients of the cost function $J$ with respect to the Fourier coefficients $a, b$ as follows:

$$
\frac{\partial J}{\partial a}=\frac{1}{N^{\Gamma}} \sqrt{\sum_{(m, n) \Gamma \Gamma}\left(\frac{\partial J}{\partial a_{m, n}}\right)^{2}}
$$




$$
\frac{\partial J}{\partial b}=\frac{1}{N^{\Gamma}} \sqrt{\sum_{(m, n) \in \Gamma}\left(\frac{\partial J}{\partial b_{m, n}}\right)^{2}},
$$

where $N^{\Gamma}$ represents the number of the OBCs $\Gamma$. Figure 4 shows the gradients of the cost function $J$ with respect to the Fourier coefficients $a, b$ (normalized by their first values $\frac{\partial J_{1}}{\partial a}, \frac{\partial J_{1}}{\partial b}$, where $J_{1}$ is the cost value $J$ of the first iteration) versus the number of iterations. From Figures 3-4 we can see that both the cost function and gradients drop dramatically in the optimization process, which demonstrate the strong inversion ability of this model.

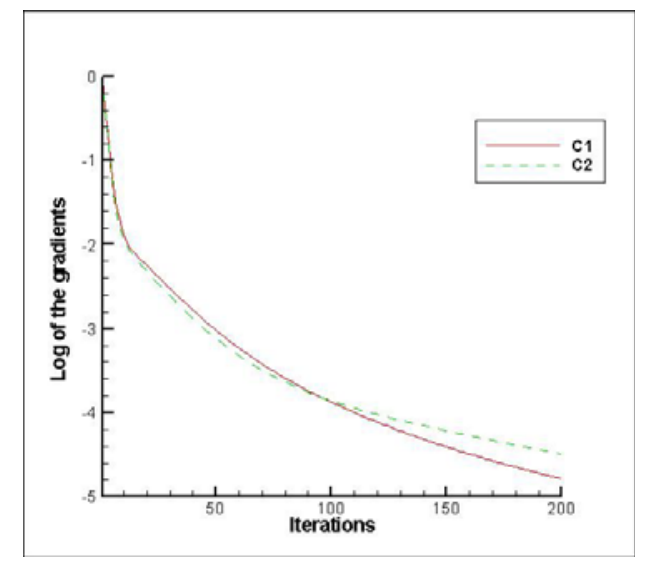

FIGURE IV. THE CURVES C1 AND C2 REPRESENT THE GRADIENTS OF $J$ WITH RESPECT TO THE FOURIER COEFFICIENTS $a$ AND $b$ OF THE OBCS VERSUS ITERATION NUMBER, RESPECTIVELY

\section{SUMMARY AND CONCLUSIONS}

4D VAR data assimilation method is widely used for the optimization of the model parameters such as the initial values, OBCs, BFC, interface friction coefficient and horizontal eddy viscosity coefficient. It has been shown that the POD 4D VAR data assimilation method can provide an efficient means of generating reduced-order formulations and alleviating the computational load and memory requirements [13]. As a preliminary feasibility study, we use the POD 4D VAR data assimilation with a $2 \mathrm{D}$ tidal model to simulate the ${ }^{\mathrm{M}_{2}}$ tide in the Bohai Sea. The numerical experiment is carried out to optimize the temporally and spatially varying open boundary conditions by assimilating $\mathrm{T} / \mathrm{P}$ altimeter data. The absolute mean difference between the model results and observations are $2.8 \mathrm{~cm}$ and $2.3^{\circ}$ for amplitude and phase-lag, respectively, suggesting that the construction of the POD reduced order model and the inversion of control variables are successful.

\section{ACKNOWLEDGMENTS}

The authors acknowledge the support of the Natural Science Foundation of Shandong Province of China through Grants ZR2014DM017 and ZR2015PF002, the National Natural Science Foundation of China through Grants 41476101 and 41371496, Qingdao Science of Technology Planning Project through Grant 13-1-4-121-jch.

\section{REFERENCES}

[1] I. M. Navon, X. Zou, J. Derber, and J. Sela, "Variational data assimilation with an adiabatic version of the NMC spectral model," Monthly Weather Review, vol. 120, no. 7, pp. 1433-14446.

[2] A. J. Zhang, E. Wei, and B. B. Parker, “Optimal estimation of tidal open boundary conditions using predicted tides and adjoint data assimilation technique," Continental Shelf Research, vol. 23, no. 11-13, pp. 10551070, 2003.

[3] A. Z. Cao, Z. Guo, and X. Q. Lv, "Inversion of two-dimensional tidal open boundary conditions of $\mathrm{M}_{2}$ constituent in the Bohai and Yellow Seas," Chinese Journal of Oceanology and Limnology, vol. 30, no. 5, pp. 868-875, 2012.

[4] V. Taillandier, V. Echevin, L. Mortier, and J. L. Devenon, "Controlling boundary conditions with a four-dimensional variational dataassimilation method in a non-stratified open coastal model," Ocean Dynamics, vol. 54, no. 2, pp. 284-298, 2004.

[5] I. Shulman, "Local data assimilation in specification of open boundary conditions," Journal of Atmospheric and Oceanic Technology, vol. 14, no. 6, pp. 1409-1419, 1997.

[6] I. Shulman, J. K. Lewis, A. F. Blumberg, and B. N. Kim, "optimized boundary conditions and data assimilation with application to the $\mathrm{M}_{2}$ tide in the yellow sea," Journal of Atmospheric and Oceanic Technology, vol. 15, no. 4, pp. 1066-1071, 1998.

[7] E. Kazantsev, "Boundary conditions control for a shallow-water model," International Journal for Numerical Methods in Fluids, vol. 68, no. 5, pp. 625-641, 2012.

[8] D. L. T. Anderson, J. Sheinbaum, and K. Haines, "data assimilation in ocean models," Reports on Progress in Physics, vol. 59, no. 10, pp 1209-1266, 1996.

[9] U. Seiler, "Estimation of open boundary conditions with the adjoint method,” Journal of Geophysical Research, vol. 98, no. C12, pp. 2285522870, 1993.

[10] X. Q. Lv and J. Zhang, "Numerical study on spatially varying bottom friction coefficient of a 2D tidal model with adjoint method," Continental Shelf Research; vol. 26, no. 16, pp. 1905-1923, 2006. Y. Yorozu, M. Hirano, K. Oka, and Y. Tagawa, "Electron spectroscopy studies on magneto-optical media and plastic substrate interface,” IEEE Transl. J. Magn. Japan, vol. 2, pp. 740-741, August 1987 [Digests 9th Annual Conf. Magnetics Japan, p. 301, 1982].

[11] J. Zhang and X. Q. Lv, "Parameter estimation for a three-dimensional numerical barotropic tidal model with adjoint method," International Journal for Numerical Methods in Fluids, vol. 57, no. 1, pp. 47-92, 2008.

[12] J. Du, J. Zhu, Z. D. Luo, and I. M. Navon, “An optimizing finite difference scheme based on proper orthogonal decomposition for CVD equations,” Communications in Numerical Methods in Engineering, vol. 27, no. 1, pp. 78-94, 2011.

[13] Y. H. Cao, J. Zhu, Z. H. Luo, and I. M. Navon, "Reduced order modelling of the upper tropical pacific ocean model using proper orthogonal decomposition," Computers and Mathematics with Applications; vol. 52, no. 8-9, pp. 1373-1386, 2006.

[14] S. G. Qian, X. Q. Lu, Y. H. Cao, and F. J. Shao, "Parameter Estimation for a 2D Tidal Model with POD 4D VAR Data Assimilation", Mathematical Problems in Engineering, Vol. 2016, Article ID 6751537, 14 pages, 2016. 